\title{
Acrodermatite entéropathique chez un garçon de 3 mois
}

\author{
Alexander K.C. Leung MD, Kin Fon Leong MD, Joseph M. Lam MD
}

Citation : CMAJ 2021 February 16;193:E243. doi : 10.1503/cmaj.201181-f

Voir la version anglaise de l'article ici : www.cmaj.ca/lookup/doi/10.1503/cmaj.201181

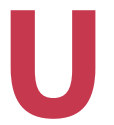

$\mathrm{n}$ garçon de 3 mois exclusivement allaité a été amené chez le médecin parce qu'il présentait, depuis 2 semaines, de la diarrhée et un érythème fessier persistant malgré l'application de crème protectrice et des changements de couche fréquents. Il est né à terme, au troisième centile, d'une mère primigeste de 23 ans, et suivait sa courbe de croissance. Ses parents n'étaient pas consanguins et n'avaient pas d'antécédents familiaux connus de maladie génétique. À l'examen physique, le garçon était irritable et présentait des plaques érythémateuses érosives, symétriques et bien délimitées accompagnées de desquamation et de croûtes sur les orteils et dans la région génitale (figure $1 \mathrm{~A}$ ), ainsi que sur les joues, le pavillon des oreilles et le cou (figure 1B). Nous lui avons diagnostiqué une acrodermatite entéropathique acquise. Ce diagnostic était appuyé par une faible concentration plasmatique de zinc $(18 \mu \mathrm{g} / \mathrm{dL}$ [plage normale $70-120 \mu \mathrm{g} / \mathrm{dL}$ ) ) et de phosphatase alcaline $(20 \mathrm{U} / \mathrm{L}$ [plage normale 96-360 U/L]). Nous avons alors commencé à lui administrer des suppléments de zinc, à raison de $0,5 \mathrm{mg} / \mathrm{kg} / \mathrm{j}$, après quoi ses symptômes et lésions cutanées se sont résorbés. Son taux de zinc est redevenu normal et l'est resté même après l'arrêt de la supplémentation.

L'acrodermatite entéropathique peut être due à une carence en zinc héréditaire ou acquise ${ }^{1}$. La forme héréditaire découle de mutations homozygotes du gène SLC39A4, qui nuisent au transport transmembranaire et à l'absorption du zinc $^{2}$. Elle survient dans 1 naissance vivante sur 500000 , sans égard au sexe ou à la race ${ }^{3}$. Généralement, les symptômes apparaissent entre 4 et 10 semaines de vie chez les enfants nourris avec de la formule pour nourrissons, et beaucoup plus tard chez ceux allaités, en raison de la biodisponibilité accrue du zinc et de la présence d'une protéine de transport en facilitant l'absorption dans le lait maternel ${ }^{1}$. Quant à la forme acquise, elle est le plus souvent causée par un régime alimentaire faible en zinc ou du lait maternel à faible teneur en ligand liant le zinc. Elle touche $2 \%$ des enfants aux États-Unis, et $20 \%$ des enfants dans les pays à revenu faible ou intermédiaire ${ }^{1}$.

Les médecins devraient mesurer le taux sérique de zinc des enfants qui présentent de l'irritabilité, de la diarrhée chronique, de l'alopécie et un érythème périorificiel, périnéal et acral. Comme un dépistage génétique n'était pas possible, nous avons présumé que le patient présentait une acrodermatite entéropathique acquise étant donné sa forte réponse à la supplémentation en zinc. En cas de carence en zinc acquise, l'amélioration de l'alimentation chez la mère et la maturation intestinale de l'enfant font souvent en sorte que le taux de zinc reste normal, même après l'arrêt de la supplémentation. 


\section{Références}

1. Leung AKC, Barankin B. Case in point: acrodermatitis enteropathica: an infant with skin lesions and diarrhea from zinc deficiency. Consultant for Pediatricians 2016;15:406-8. Accessible ici : www.consultant360.com/articles/ case-point-acrodermatitis-enteropathica (consulté le 15 janv. 2021).

2. Del Ciampo IRL, Sawamura R, Del Ciampo LA, et al. Acrodermatitis enteropathica: clinical manifestations and pediatric diagnosis [article en portugais]. Rev Paul Pediatr 2018;36:238-41.

3. Nistor N, Ciontu L, Frasinariu O-E, et al. Acrodermatitis enteropathica: a case report. Medicine (Baltimore) 2016;95:e3553.

Les images cliniques sont choisies pour leur caractère particulièrement intéressant, classique ou impressionnant. Toute soumission d'image de haute résolution claire et bien identifiée doit être accompagnée d'une légende aux fins de publication. On demande aussi une brève explication (300 mots maximum) de la portée éducative des images, et des références minimales. Le consentement écrit du patient au regard de la publication doit être obtenu avant la soumission.
Intérêts concurrents : Aucun déclaré.

Cet article a été révisé par des pairs.

Les auteurs ont obtenu le consentement des parents.

Affiliations : Université de Calgary (Leung), Hôpital pour enfants de l'Alberta (Leung), Calgary, Alb.; Institut de pédiatrie (Leong), Hôpital général de Kuala Lumpur, Kuala Lumpur, Malaisie; Département de dermatologie et des sciences dermatologiques (Lam), Université de la Colombie-Britannique, Hôpital pour enfants de la ColombieBritannique (Lam), Vancouver, C.-B.

Propriété intellectuelle du contenu : Il s'agit d'un article en libre accès distribué conformément aux modalités de la licence Creative Commons Attributions (CC BY-NC-ND 4.0), qui permet l'utilisation, la diffusion et la reproduction dans tout médium à la condition que la publication originale soit adéquatement citée, que l'utilisation se fasse à des fins non commerciales (c.-à-d. recherche ou formation) et qu'aucune modification ni adaptation n'y soit apportée. Voir : https://creativecommons.org/licenses/by-nc-nd/4.0/deed.fr.

Correspondance : Alexander Leung, aleung@ucalgary.ca 